\title{
Testing of Device from Other Lot/Batch Returned from User
}

National Cancer Institute

\section{Source}

National Cancer Institute. Testing of Device from Other Lot/Batch Returned from User.

NCI Thesaurus. Code C139449.

The investigation employed relevant empirical testing of the device of another lot or batch than that of the suspected device in the reported adverse event in order to support the identification of possible causes for the adverse event. This includes devices without a lot/batch designation. The device was returned from the user. Relevant testing would typically be based on test methods used for evaluating safety and performance as described in the technical file. Relevant testing would typically be based on test methods used for evaluating safety and performance as described in the latest relevant standards. 